\title{
Verzeichnis der Autor:innen
}

\author{
Albrecht, Rita \\ https://orcid.org/0000-0003-0772-1057 \\ Rita Albrecht wurde für den gehobenen Bibliotheksdienst an der Stadt- und \\ Universitätsbibliothek und der Bibliotheksschule Frankfurt ausgebildet. Seit \\ 1998 ist sie in der hebis-Verbundzentrale tätig, ist dort Leiterin der Gruppe \\ Metadaten und Standardisierung sowie zuständig für die Öffentlichkeitsarbeit.
}

\section{Baierer, Konstantin}

https://orcid.org/0000-0003-2397-242X

Konstantin Baierer studierte Bibliotheks- und Informationswissenschaft und Informatik an der Humboldt-Universität zu Berlin und arbeitete 2012-2014 am Projekt DM2E (Digital Manuscripts to Europeana) in Berlin, 2014-2016 in Mannheim am Projekt InFoLiS (Integration von Forschungsdaten und Literatur) sowie 2016-2018 für die Universitätsbibliothek Heidelberg an digitalen Editionen. Seit 2018 ist er an der Staatsbibliothek zu Berlin für die Interoperabilität der Komponenten des Projekts OCR-D verantwortlich.

\section{Balakrishnan, Umamaheswari}

https://orcid.org/0000-0002-8132-9574

Uma Balakrishnan hält den Master of Science with Merit in Information and Library Studies der Robert Gorden University, Aberdeen. In der Verbundzentrale (VZG) des Gemeinsamen Bibliotheksverbundes (GBV) leitet sie u. a. das VZGProjekt coli-conc. Ihr Zuständigkeitsbereich umfasst Wissensorganisationssysteme und dazugehörige relevante Projekte.

\section{Beck, Cyrus, Dr.}

https://orcid.org/0000-0002-5719-2651

Cyrus Beck ist stellvertretender Leiter der Abteilung für Informationskompetenzvermittlung, Digitale Dienste und Entwicklung sowie Fachreferent für Politik- und Militärwissenschaft an der Zentralbibliothek Zürich. Er bekleidete die ehemalige Kompetenzstelle für Normdaten in der Inhaltserschließung des Informationsverbunds Deutschschweiz (IDS) und war bis 2019 Mitglied des Expertenteams RDA-Anwendungsprofil für die verbale Inhaltserschließung. 


\section{Beckmann, Regine}

https://orcid.org/0000-0001-7052-2793

Regine Beckmann ist seit 2005 an der Staatsbibliothek zu Berlin - Preußischer Kulturbesitz beschäftigt. Zunächst betreute sie die Fachreferate Kunst, Architektur, Theater und Film, seit 2014 koordiniert sie als Stabsstelle den Bereich Inhaltserschließung. In dieser Funktion vertritt sie die Bibliothek in verschiedenen überregionalen Fachgremien, unter anderem in der Fachgruppe Erschließung und im Expertenteam RDA-Anwendungsprofil für die verbale Inhaltserschließung an der DNB sowie in der für den SWB und den GBV gemeinsam eingerichteten AG K10plus Sacherschließung. Im GBV ist sie Sprecherin der Facharbeitsgruppe Erschließung und Informationsvermittlung und Mitglied des Fachbeirates. Von 2016-2020 unterrichtete sie als Lehrbeauftragte im Master-Fernstudiengang des Instituts für Bibliotheks- und Informationswissenschaft der Humboldt-Universität zu Berlin die Grundlagen verbaler und klassifikatorischer Informationsaufbereitung.

\section{Block, Barbara, Dr.}

https://orcid.org/0000-0002-7486-3189

Barbara Block studierte Geschichte und Mathematik und absolvierte danach eine Ausbildung für den Höheren Bibliotheksdienst an der FHBD Köln. Sie ist bei der Verbundzentrale des GBV in Göttingen als Leiterin der Abteilung Bibliothekarische Dienste tätig.

\section{Bourgonje, Peter}

https://orcid.org/0000-0003-3541-0678

Peter Bourgonje hat einen Bachelor in Linguistik (2007) und einen Master in Computerlinguistik (2010), beide von der Universität Utrecht (Niederlande). Nach Tätigkeiten als Computerlinguist in verschiedenen Unternehmen kam er 2015 als wissenschaftlicher Mitarbeiter an das Deutsche Forschungszentrum für Künstliche Intelligenz (DFKI; Forschungsbereich Speech and Language Technology) in Berlin. Zusätzlich war Peter Bourgonje von 2017 bis 2020 als Doktorand im Bereich Diskursparsing an der Universität Potsdam tätig. Die Verteidigung der Dissertation ist für Anfang 2021 vorgesehen.

\section{Expertenteam RDA-Anwendungsprofil für die verbale Inhaltserschließung}

Das Expertenteam RDA-Anwendungsprofil für die verbale Inhaltserschließung besteht aus den Personen Cyrus Beck (bis Ende 2019), Regine Beckmann, Madeleine Boxler (bis Ende 2020), Michael Franke-Maier, Urs Frei, Werner Holbach, Ar- 
min Kühn, Bettina Kunz, Julijana Nadj-Guttandin, Sarah Pielmeier, Esther Scheven, Christoph Steiner, Heidrun Wiesenmüller und Barbara Wolf-Dahm.

\section{Franke-Maier, Michael}

https://orcid.org/0000-0003-4263-2754

Michael Franke-Maier ist stellvertretender Leiter der Zugangsabteilung der Universitätsbibliothek der Freien Universität Berlin, leitet dort den Bereich Sacherschließung und ist für die Koordination der Inhaltserschließung im Bibliothekssystem der FU verantwortlich. Zudem vertritt er auf nationaler Ebene den Kooperativen Bibliotheksverbund Berlin-Brandenburg (KOBV) im GND-Ausschuss und in diversen nachgeordneten Gremien, wie dem Expertenteam RDAAnwendungsprofil für die verbale Inhaltserschließung (ET RAVI). Im Zusammenhang mit der strategischen Allianz zwischen KOBV und dem Bibliotheksverbund Bayern ist er seit Sommer 2014 stellvertretender Vorsitzender der Arbeitsgruppe für Sacherschließung der Kommission für Erschließung und Metadaten. Weiterhin ist er seit Ende 2016 Mitglied im Beirat der Regensburger Verbundklassifikation.

\section{Frei, Urs, Dr.}

Urs Frei absolvierte ab 1977 ein Studium und Doktorat in Chemie an der ETH Zürich, zwischen 1992-1996 arbeitete er in der Hauptbibliothek der ETH Zürich, ab 1996 war er im Dienst Sacherschliessung der Schweizerischen Nationalbibliothek in Bern tätig, ab 1997 als stellvertretender Leiter. Ab 1998 arbeitet er in Expertengremien für Inhaltserschliessung des Standardisierungsausschusses (u. a. ET RAVI) mit.

\section{Gerber, Mike}

https://orcid.org/0000-0003-2167-4322

Mike Gerber hat Informatik am Karlsruher Institut für Technologie studiert und ist seit 2019 im Rahmen des QURATOR-Projekts an der Staatsbibliothek zu Berlin beschäftigt. Nach einem Exkurs in der Startup-Szene beschäftigt er sich heute mit der Optical Character Recognition von historischen Drucken. Sein Hauptinteresse gilt dem Machine Learning und der OCR.

\section{Golub, Koraljka, Prof. Dr.}

https://orcid.org/0000-0003-4169-4777

Koraljka Golub leitet die iSchool der Linnaeus-Universität, das iInstitute, und ist Ko-Leiterin der Digital Humanities Initiative an der Linnaeus-Universität. 
Ihre Forschungsschwerpunkte sind Digitale Bibliotheken und Information Retrieval. Dabei liegt ihr spezieller Fokus auf Themen der Wissensorganisation, der Integration von Wissensorganisationssystemen in Social Tagging bzw. automatisierte Verschlagwortung sowie der Evaluierung des daraus resultierenden Information Retrievals durch Endnutzende.

\section{Holbach, Werner}

https://orcid.org/0000-0002-2731-6967

Nach dem Studium der Elektrotechnik an der Universität Kaiserslautern und dem anschließenden Bibliotheksreferendariat war Werner Holbach von 1988 bis 2001 als wissenschaftlicher Bibliothekar an der UB der TU München tätig; seit 2001 an der Bayerischen Staatsbibliothek. Zurzeit ist er als Referatsleiter Länder- und Fachreferate verantwortlich für Sacherschließung, Erwerbung und Normdaten. Er ist Vorsitzender der Arbeitsgruppe für Sacherschließung der Kommission für Erschließung und Metadaten (BVB/KOBV) und ist Mitglied im Expertenteam RDA-Anwendungsprofil für die verbale Inhaltserschließung sowie in der Fachgruppe Erschließung und im GND-Ausschuss.

\section{Junger, Ulrike}

Ulrike Junger studierte die Fächer Evangelische Theologie und Psychologie und absolvierte in den Jahren 1992-1994 ihr Bibliotheksreferendariat. Zwischen 1995 und 2009 hatte sie verschiedene Positionen an der SUB Göttingen, der Verbundzentrale des GBV und der Staatsbibliothek zu Berlin inne. Seit 2009 ist sie an der Deutschen Nationalbibliothek, derzeit als Leiterin des Fachbereichs Erwerbung und Erschließung.

\section{Kasprzik, Anna, Dr.} https://orcid.org/0000-0002-1019-3606

Anna Kasprzik hat Formale Linguistik, Informatik und Kognitionspsychologie studiert und in der Theoretischen Informatik promoviert. Danach hat sie am KIM Konstanz und an der Bibliotheksakademie in München das Bibliotheksreferendariat abgelegt und eine Weile für den Bibliotheksverbund Bayern gearbeitet. Anschließend hat sie sich in der Forschung und Entwicklung der TIB Hannover mit Wissensorganisationssystemen (Thesauri und Ontologien) befasst. Seit 2018 koordiniert sie an der ZBW - Leibniz-Informationszentrum Wirtschaft die Automatisierung der Sacherschließung mit Machine-Learning-Methoden 
aus der hausinternen angewandten Forschung und deren Verzahnung mit semantischen Technologien.

\section{Király, Péter, Ph. D.}

https://orcid.org/0000-0002-8749-4597

Péter Király ist Softwareentwickler und Forscher auf dem Gebiet des kulturellen Erbes und der digitalen Geisteswissenschaften. Sein Forschungsgebiet ist die Messung der Qualität von Metadaten. Er ist Mitglied der Göttinger eResearch Alliance, einem Gemeinschaftsprojekt des Rechenzentrums und der Bibliothek des Göttingen Campus. Seine Hauptverantwortung ist GRO.data, ein auf Dataverse basierendes Tool zur Veröffentlichung von Forschungsdaten. Er ist aktiver Mitarbeiter verschiedener Open-Source-Projekte.

\section{Kratzer, Mathias, Dr.}

https://orcid.org/0000-0003-3470-0622

Mathias Kratzer studierte Mathematik und Informatik an der Technischen Universität München. Nach seiner Promotion im Bereich Darstellungstheorie endlicher einfacher Gruppen an der Universität Duisburg-Essen nahm er 2003 seine Tätigkeit an der Bayerischen Staatsbibliothek in der Verbundzentrale des Bibliotheksverbundes Bayern auf. Von 2013 bis 2021 leitete er dort das Referat Verbundnahe Dienste. Seit August 2021 leitet er das Stabsreferat Informationstechnologie der Bayerischen Staatsbibliothek.

\section{Kühn, Armin}

Armin Kühn ist seit 1995 im Bibliotheksservice-Zentrum Baden-Württemberg als Bibliothekar beschäftigt und dort $\mathrm{u}$. a. für die verbale und klassifikatorische Sacherschließung zuständig. Mitarbeit im überregionalen Expertenteam RDAAnwendungsprofil für die verbale Inhaltserschließung, zuvor Mitglied der Expertengruppe Sacherschließung.

\section{Kunz, Bettina}

Bettina Kunz leitet die Zentralredaktion Sacherschließung des Gemeinsamen Bibliotheksverbundes (GBV) und betreut die Teilnehmerbibliotheken in allen Belangen der kooperativen Sacherschließung im GBV. Auf überregionaler Ebene vertritt sie den GBV im Bereich Inhaltserschließung im Expertenteam RDA-Anwendungsprofil für die verbale Inhaltserschließung (ET RAVI) der Fachgruppe Erschließung des Standardisierungsausschusses und dem Expertenteam Werke des GND-Ausschusses. 


\section{Labusch, Kai, Dr.}

https://orcid.org/0000-0002-7275-5483

Kai Labusch, Studium der Informatik mit Nebenfach Biomathematik an der Universität zu Lübeck, ist seit 2019 Mitarbeiter im QURATOR-Team der Staatsbibliothek zu Berlin. Nach seiner Promotion zum Thema Sparse Coding am Institut für Neuro- und Bioinformatik der Universität zu Lübeck entwickelte er Anwendungen maschineller Lernverfahren unter anderem zur Sprachverarbeitung und in der Stahlindustrie. Schwerpunkte seiner aktuellen Forschungsinteressen sind Unüberwachtes Lernen und Transferlernen in neuronalen Netzen.

\section{Laczny, Joachim, Dr.}

https://orcid.org/0000-0001-5629-2557

Joachim Laczny studierte Geschichtswissenschaft, Geografie, Volkskunde und Hochschuldidaktik an der Universität Hamburg und promovierte mit dem Schwerpunkt in spätmittelalterlicher Wirtschaftsgeschichte. Das Bibliotheksreferendariat absolvierte er an der Niedersächsischen Staats- und Universitätsbibliothek Göttingen und der Bibliotheksakademie Bayern in München. Er ist an der Staatsbibliothek zu Berlin, Preußischer Kulturbesitz, in der Abteilung Bestandsaufbau - Stabsstelle Metadatenmanagement - tätig.

\section{Ledl, Andreas, Dr.}

https://orcid.org/0000-0002-0629-0446

Andreas Ledl leitet die Campus Muttenz Bibliothek der Fachhochschule Nordwestschweiz (FHNW). Zuvor war er Fachreferent an den Universitätsbibliotheken Basel und Bern und von 2018-2020 Vizepräsident der International Society for Knowledge Organization (ISKO). 2013 gründete er das Basic Register of Thesauri, Ontologies and Classifications (BARTOC.org).

\section{Leitner, Elena}

https://orcid.org/0000-0002-6363-4807

Elena Leitner erhielt ihren Bachelorabschluss in Computerlinguistik (2019) an der Universität Potsdam. Seit 2018 ist Elena Leitner im Forschungsbereich Speech and Language Technology am Deutschen Forschungszentrum für Künstliche Intelligenz (DFKI) in Berlin tätig - zunächst als wissenschaftliche Hilfskraft im EU-Projekt Building the Legal Knowledge Graph for Smart Compliance Services in Multilingual Europe (Lynx) und seit Mitte 2019 als wissenschaftliche Mitarbeiterin im DFG-Projekt Interfaces to Data for Historical Social Network Analysis (SoNAR (IDH)). 


\section{Maas, Jan Frederik, Dr.-Ing.}

https://orcid.org/0000-0002-6374-7414

Jan Frederik Maas hat Computerlinguistik studiert und in angewandter Informatik promoviert. Seit seinem erfolgreichen Abschluss eines Referendariats an der TIB Hannover arbeitet er als Fachreferent, IT-Projektleiter und Softwareentwickler an der Staats- und Universitätsbibliothek Hamburg. Er ist Sprecher des beluga-core-Entwicklungsverbundes mehrerer Hochschulbibliotheken, der modulare Erweiterungen für die quelloffene Discovery-Software VuFind zur Verfügung stellt.

\section{Menzel, Sina}

https://orcid.org/0000-0003-1798-2672

Sina Menzel war im Projekt SoNAR für die Evaluierung und Qualitätssicherung zuständig und ist seit Herbst 2020 verantwortlich für die Benutzungsforschung an der Universitätsbibliothek der Freien Universität Berlin.

\section{Moreno Schneider, Julián, Dr.}

https://orcid.org/0000-0003-1418-9935

Julián Moreno Schneider ist als Senior Researcher im Forschungsbereich Speech and Language Technology im Deutschen Forschungszentrum für Künstliche Intelligenz (DFKI) in Berlin tätig. Er hat einen Bachelor in Telekommunikationstechnik, einen Master in Informatik und einen $\mathrm{PhD}$, ebenfalls in Informatik, mit dem Schwerpunkt Multimedia Information Retrieval (alle von der Universidad Carlos III de Madrid, Spanien). Er war bzw. ist in verschiedenen nationalen (Buscamedia, Bravo, SOPAT, DKT, QURATOR, SPEAKER) und internationalen (Lynx, ELG) Forschungsprojekten tätig. Aktuell beschäftigt er sich insbesondere in den Projekten QURATOR, Lynx und SPEAKER mit der Entwicklung von Plattformen und Backend-Technologien.

\section{Nadj-Guttandin, Julijana, Dr.}

https://orcid.org/0000-0002-5157-7374

Julijana Nadj-Guttandin arbeitet in der Abteilung Inhaltserschließung der Deutschen Nationalbibliothek am Standort Frankfurt. Sie studierte Anglistik, Russistik und Politikwissenschaften an den Universitäten Gießen und Loughborough, UK. Nach der Promotion absolvierte sie das Referendariat für den höheren Bibliotheksdienst an der Universitätsbibliothek Marburg, verbunden mit dem Fernstudium am Institut für Bibliotheks- und Informationswissenschaft der Humboldt-Universität zu Berlin. Seit 2008 ist sie als Fachreferentin in der Abtei- 
lung Inhaltserschließung der Deutschen Nationalbibliothek beschäftigt. Sie betreut verschiedene geistes- und sozialwissenschaftliche Fächer, ist mitverantwortlich für die Regelwerksarbeit zur verbalen Erschließung und Mitglied im Expertenteam RDA-Anwendungsprofil für die verbale Inhaltserschließung.

\section{Neudecker, Clemens}

https://orcid.org/0000-0001-5293-8322

Clemens Neudecker, Studium der Philosophie, Informatik und Politischen Wissenschaften an der Ludwig-Maximilians-Universität München, ist seit 2014 als Projektkoordinator und Forschungsreferent in der Generaldirektion der Staatsbibliothek zu Berlin beschäftigt. Nach seiner Tätigkeit als wissenschaftlicher Mitarbeiter im Digitalisierungszentrum der Bayerischen Staatsbibliothek von 2003-2009 war er 2009-2014 Projektleiter in der Forschungsabteilung der Nationalbibliothek der Niederlande. Sein Hauptinteresse gilt der Forschung und Entwicklung in den Bereichen Computer Vision, Natural Language Processing sowie den Digital Humanities.

\section{Ostendorff, Malte}

https://orcid.org/0000-0003-2077-9907

Malte Ostendorff ist als wissenschaftlicher Mitarbeiter im Forschungsbereich Speech and Language Technology im Deutschen Forschungszentrum für Künstliche Intelligenz (DFKI) in Berlin tätig. Daneben promoviert Malte Ostendorff an der Universität Konstanz zum Thema Dokumentenähnlichkeit. Als Gründer und Vorstandsmitglied des Open Justice e. V. setzt er sich für einen transparenten Rechtsstaat ein.

\section{Pielmeier, Sarah}

https://orcid.org/0000-0001-9050-9895

Sarah Pielmeier, Magistra der Soziologie, arbeitete seit 2000 nach einer Ausbildung zur Bibliothekssekretärin mit Unterbrechungen in mehreren Bibliotheken. Seit 2014 arbeitet sie in der Universitäts- und Landesbibliothek Münster (ULB) in wechselnden Aufgabenbereichen und schloss im Jahr 2020 ihren Master in Library and Information Science an der Technischen Hochschule Köln ab. Derzeit leitet Sie die Abteilung Sacherschließung an der ULB und ist Mitglied im Expertenteam RDA-Anwendungsprofil für die verbale Inhaltserschließung. 


\section{Pintscher, Lydia}

https://orcid.org/0000-0002-3939-2115

Lydia Pintscher ist die Produktmanagerin für Wikidata und arbeitet für Wikimedia Deutschland. Sie studierte Informatik an der Universität Karlsruhe mit einem Schwerpunkt in Medizin und Linguistik. Sie ist außerdem seit über 15 Jahren in verschiedenen Freien Softwareprojekten tätig, hauptsächlich als Vorstandsmitglied des KDE e. V.

\section{Peters, Stefan}

https://orcid.org/0000-0002-4087-8227

Stefan Peters arbeitet als Softwareentwickler bei der Verbundzentrale des GBV (VZG) in Göttingen. Er ist primär für die Entwicklung der Infrastruktur des coliconc-Projektes und des Open Source Mapping-Tools Cocoda zuständig.

\section{Petras, Vivien, Prof. Dr.}

https://orcid.org/0000-0002-8113-1509

Vivien Petras studierte Bibliotheks- und Informationswissenschaft, BWL und Kunstgeschichte an der Humboldt-Universität zu Berlin. Promotion (2006) an der University of California, Berkeley, USA, in Information Management and Systems. Stellv. Abteilungsleiterin der Abteilung Forschung und Entwicklung des Informationszentrums Sozialwissenschaften, Bonn (jetzt GESIS). Seit 2009 Professorin für Information Retrieval, Institut für Bibliotheks- und Informationswissenschaft der Humboldt-Universität zu Berlin. Ihre Forschungsinteressen fokussieren auf das mehrsprachige und interaktive Information Retrieval, die Qualität und Heterogenität von Metadaten in Kulturerbeinformationssystemen sowie domänenspezifische Wissensorganisation.

\section{Pohl, Adrian}

https://orcid.org/0000-0001-9083-7442

Adrian Pohl leitet die Gruppe Offene Infrastruktur des Hochschulbibliothekszentrums des Landes Nordrhein-Westfalen (hbz), die sich mit den verschiedenen Ebenen von Publikation, Austausch und Management bibliothekarischer Daten im Web befasst. Er ist Co-Vorsitzender des Programmkomitees der SWIB-Konferenz (Semantic Web in Bibliotheken). Er hat Abschlüsse in Kommunikationswissenschaft und Philosophie von der RWTH Aachen sowie in Bibliotheks- und Informationswissenschaft von der TH Köln. 


\section{Rehm, Georg, Prof. Dr.}

https://orcid.org/0000-0002-7800-1893

Georg Rehm ist als Principal Researcher im Forschungsbereich Speech and Language Technology am Deutschen Forschungszentrum für Künstliche Intelligenz (DFKI) in Berlin tätig. Er ist Koordinator des BMBF-Projekts QURATOR (Curation Technologies) und des EU-Projekts European Language Grid (ELG) sowie Ko-Koordinator des EU-Projekts European Language Equality (ELE). Daneben ist er seit 2010 - Generalsekretär des EU-Exzellenznetzwerks META-NET und leitet seit 2013 das deutsch-österreichische Chapter des World Wide Web Consortiums (W3C). Ferner ist Georg Rehm aktuell in die Projekte Lynx (EU), HumanE-AI-Net (EU), SPEAKER (BMWi), SoNAR (DFG) und PANQURA (BMBF) involviert. 2018 wurde Georg Rehm der Ehrentitel DFKI Research Fellow verliehen.

\section{Sack, Harald, Prof. Dr.}

https://orcid.org/0000-0001-7069-9804

Harald Sack ist Professor für Information Service Engineering am FIZ Karlsruhe - Leibniz-Institut für Informationsinfrastruktur und Karlsruher Institut für Technologie (KIT). Nach dem Studium der Informatik an der Universität der Bundeswehr München wurde er 1997 assoziiertes Mitglied des Graduiertenprogramms Mathematische Optimierung an der Universität Trier und promovierte 2002 in Informatik. Nach seiner Zeit als PostDoc an der Friedrich-Schiller-Universität in Jena leitete er von 2009 bis 2016 die Forschungsgruppe Semantische Technologien und Multimedia Retrieval am Hasso-Plattner-Institut für IT-Systems Engineering an der Universität Potsdam. Seine aktuellen Forschungsgebiete umfassen semantische Technologien, Knowledge Discovery sowie hybride Anwendungen symbolischer und subsymbolischer Künstlicher Intelligenz. Er war als General Chair, Program Committee Chair und (Senior) Program Committee Member bei zahlreichen internationalen Konferenzen und Workshops tätig. Harald Sack hat mehr als 150 wissenschaftliche Arbeiten in internationalen Fachzeitschriften und auf Konferenzen veröffentlicht, darunter mehrere Standardlehrbücher.

\section{Scheven, Esther}

Esther Scheven, wissenschaftliche Bibliothekarin, Diplom-Bibliothekarin, Diplom-Geologin und Diplom-Biologin, arbeitet seit 1995 in der Deutschen Nationalbibliothek als Fachreferentin in der Abteilung Inhaltserschließung. Bis 2016 hat sie die Expertengruppe Sacherschließung und die Überarbeitung der 4. Auflage der RSWK (2017) geleitet, ist heute Mitglied im Expertenteam RDA-Anwen- 
dungsprofil für die verbale Inhaltserschließung. Sie gehörte zur Projektleitung des GND-Projekts (2009-2012).

\section{Schnaitter, Hannes}

https://orcid.org/0000-0002-1602-6032

Hannes Schnaitter studierte Bibliotheks- und Informationswissenschaft (B. A.) und Information Science (M. A.) an der Humboldt-Universität zu Berlin. Er ist im SoNAR-Projekt für die Qualitätssicherung und Evaluierung zuständig.

\section{Scholze, Frank}

https://orcid.org/0000-0003-3404-1452

Frank Scholze ist seit 2020 Generaldirektor der Deutschen Nationalbibliothek. Er studierte Bibliothekswesen an der Hochschule der Medien Stuttgart, sowie Kunstgeschichte und Anglistik an der Universität Stuttgart. Danach war er in verschiedenen Projekten im Bereich digitaler Bibliotheken, sowie als Fachreferent und Leiter der Benutzungsabteilung an der Universitätsbibliothek Stuttgart tätig. Nach zweijähriger Tätigkeit im Ministerium für Wissenschaft, Forschung und Kunst Baden-Württemberg übernahm er im Januar 2010 die Leitung der KIT-Bibliothek. 2016 wurde er in den Bundesvorstand des Deutschen Bibliotheksverbandes (dbv) gewählt und 2019 wiedergewählt.

\section{Schürmann, Hans, Dr.}

https://orcid.org/0000-0003-2532-3896

Hans Schürmann ist seit 2005 an der Zentral- und Hochschulbibliothek Luzern in verschiedenen Funktionen tätig. Heute beschäftigt er sich einerseits im Bereich E-Science mit Repositoriumsdienstleistungen und digitaler Langzeitarchivierung und andererseits im Bereich Metadatenmanagement mit verbaler und klassifikatorischer Inhaltserschließung. Seit Februar 2021 ist er für die Swiss Library Service Platform (SLSP) Mitglied im GND-Ausschuss.

\section{Steeg, Fabian}

https://orcid.org/0000-0001-8829-1989

Fabian Steeg ist Softwareentwickler mit Schwerpunkt Informationssysteme und Open-Source-Software und arbeitet im Bereich Webentwicklung und Datenverarbeitung in der Gruppe Offene Infrastruktur am Hochschulbibliothekszentrum des Landes Nordrhein-Westfalen (hbz). Er ist Mitglied der W3C Entity Reconciliation Community Group und seit 2020 deren Co-Chair. Er hat einen Abschluss 
in Informationsverarbeitung, Allgemeiner Sprachwissenschaft und Geografie von der Universität zu Köln.

\section{Steiner, Christoph, Dr.}

Christoph Steiner ist Leiter der Abteilung Sacherschließung an der Österreichischen Nationalbibliothek, Vortragender im Universitätslehrgang Library and Information Sciences zu Themen der Inhaltserschließung, automatischer Indexierung, Mitglied diverser Fachgremien im Bereich der Inhaltserschließung, z. B. in der Zentralredaktion des Österreichischen Bibliothekenverbund, im Expertenteam RDA-Anwendungsprofil für die verbale Inhaltserschließung u. a.m.

\section{Thiessen, Peter}

https://orcid.org/0000-0002-3010-6338

Peter Thiessen ist nach einem Studium der Geisteswissenschaften sowie dem Bibliotheksreferendariat in Berlin und München seit 2014 Mitarbeiter an der Bayerischen Staatsbibliothek/Verbundzentrale des Bibliotheksverbundes Bayern und seit 2019 stellvertretender Leiter des Referats Verbundnahe Dienste.

\section{Ungváry, Rudolf}

https://orcid.org/0000-0002-8044-4319

Rudolf Ungváry hat zehn Jahre Industrieerfahrung als Maschinenbauingenieur. Seit 1974 redigierte er 40 Thesauri für Informationsinstitutionen, später Forschungsarbeiten auf dem Gebiet der Wissensorganisation. In den 1980er Jahren war er Mitglied der Deutschen Gesellschaft für Klassifikation und Mitarbeiter des Instituts für Bibliothekswissenschaft und seit 1979 führender Forschungsmitarbeiter der Ungarischen Nationalbibliothek. Nach 2000 widmete er sich der Erstellung automatisierbarer Begriffshierarchien (Ontologien) und Namensfelder (Namensräume). Derzeit ist er Mitarbeiter der Nationalbibliothek und des Digitalen Geisteswissenschaftlichen Zentrums des Museums für Literatur. Er ist Verfasser von 6 Fachbüchern und 136 Studien.

\section{Voß, Jakob, Dr.}

https://orcid.org/0000-0002-7613-4123

Jakob Voß arbeitet in Forschung und Entwicklung an der Verbundzentrale des GBV (VZG). 2013 promovierte er an der Humboldt Universität zu Berlin zu allgemeinen Strukturen in Daten. Derzeit ist er für die technische Koordination im Projekt coli-conc verantwortlich. 


\section{Wartena, Christian, Prof. Dr.}

https://orcid.org/0000-0001-5483-1529

Christian Wartena studierte Computerlinguistik in Nijmegen (Niederlande) und Potsdam, wo er 1999 promovierte. Er arbeitete für Unternehmen in Heidelberg und Enschede (Niederlande) im Bereich der automatischen Übersetzung und des Wissenstransfers. Seit 2011 ist er Professor für Sprach- und Wissensverarbeitung an der Hochschule Hannover und lehrt hier im Bachelor Informationsmanagement sowie im Master Data Analytics an der Universität Hildesheim. Er leitet verschiedene Projekte zu Sprachverarbeitung, Textanalyse und Information Retrieval. Seit 2020 ist Christian Wartena Sprecher des Forschungsclusters Smart Data Analytics.

\section{Wiesenmüller, Heidrun, Prof.}

https://orcid.org/0000-0002-9817-5292

Heidrun Wiesenmüller studierte Mittlere Geschichte, Anglistik und Mittellatein in Erlangen und Newcastle upon Tyne. Seit 2006 lehrt sie formale und inhaltliche Erschließung an der Hochschule der Medien in Stuttgart, davor war sie Fachreferentin an der Württembergischen Landesbibliothek. Sie ist Mitglied verschiedener regionaler und überregionaler Expertengremien.

\section{Wolf-Dahm, Barbara}

Barbara Wolf-Dahm betreut in der Universitätsbibliothek Augsburg die Fachreferate Theologie, Klassische Philologie und Pädagogik und ist stellvertretende Abteilungsleiterin der Abteilung Medienbearbeitung. Im Bibliotheksverbund Bayern leitet sie den in Augsburg angesiedelten Bereich der Schlagwort-Verbundredaktion. Darüber hinaus ist sie Mitglied in den überregionalen bibliothekarischen Gremien Fachgruppe Erschließung, GND-Ausschuss und Expertenteam RDA-Anwendungsprofil für die verbale Inhaltserschließung.

\section{Zaczynska, Karolina}

https://orcid.org/0000-0002-5395-5463

Karolina Zaczynska erhielt 2016 ihre Bachelorabschlüsse in Theaterwissenschaft sowie Westslawistik an der Universität Leipzig und 2018 ihren Masterabschluss im Fach Computerlinguistik und Texttechnologie an der Justus-Liebig-Universität Gießen. Nach einer Tätigkeit als wissenschaftliche Hilfskraft beim Campuslab Digitization and Computational Analytics an der Georg-August-Universität Göttingen kam Karolina Zaczynska 2019 an das Deutsche Forschungszentrum für Künstliche Intelligenz (DFKI) in Berlin, wo sie im Forschungsbe- 
reich Speech and Language Technology als wissenschaftliche Mitarbeiterin im Projekt QURATOR tätig ist.

\section{Zinck, Josefine}

https://orcid.org/0000-0002-7511-098X

Josefine Zinck, studentische Hilfskraft im SoNAR-Projekt, ist in der Evaluation maßgeblich an der intellektuellen Kontrolle der maschinellen Verfahren beteiligt. 